\title{
Identification of Pathogens from the Upper Respiratory Tract of Adult Emergency Department Patients at High Risk for Influenza Complications
}

\author{
Justin Hardick ${ }^{1}$, Kathryn Shaw-Saliba ${ }^{1}$, Breana McBryde ${ }^{1}$, charlotte gaydos $^{2}$, Yu-Hsiang \\ $\mathrm{Hsieh}^{3}$, and Richard Rothman ${ }^{1}$ \\ ${ }^{1}$ Johns Hopkins Medicine School of Medicine \\ ${ }^{2}$ Johns Hopkins University School of Medicine \\ ${ }^{3}$ Affiliation not available
}

August 22, 2020

\begin{abstract}
Acute upper respiratory infections (URIs) represent a major source of annual emergency department (EDs) visits in the United States. However, the definitive etiology of symptoms is generally not determined as testing has historically been prioritized for influenza virus and recently, respiratory syncytial virus (RSV). To elucidate the prevalence, rates of co-infections, and etiologic composition of URIs from symptomatic adult ED patients, we evaluated specimens from four geographically diverse EDs in the United States from 2013-2014 utilizing a multiplex molecular diagnostic assay. $1941 \mathrm{ED}$ patients who had signs and/or symptoms of an acute URI and were considered 'high-risk' for influenza related complications according to CDC criteria, were consecutively enrolled and tested for influenza; influenza prevalence was 9.4\% (183/1941). Among them, 799 nasopharyngeal swab specimens with sufficient residual volumes were subsequently tested for additional respiratory pathogens. The overall positivity rate was $30.1 \%(241 / 799)$, of which $6.6 \%(16 / 241)$ were co-infected. Non-influenza pathogens from most to least common were: rhinovirus/enterovirus, coronavirus, human metapneumovirus and RSV, respectively. The ratio of co-infection to mono-infection was highest amongst those with adenovirus, versus mon-infections (2.0). Broad differences in disease prevalence and pathogen distributions were observed across geographic regions; the site with the highest detection rate (for both mono and co-infections) demonstrated the greatest pathogen diversity. Adult ED patients at high-risk for influenza complications were infected with a variety of respiratory pathogens and geographic variations in the disease prevalence and co-pathogen type were observed. Further research is required to evaluate the clinical relevance of these findings.
\end{abstract}

\section{Keywords}

Emergency Department, Influenza, Multiplex Diagnostics, Respiratory Infection, Respiratory Virus

\section{Background}

Acute upper respiratory tract infections (URIs) that are caused by a diverse range of viral and bacterial pathogens are one of the most common illnesses observed in humans. ${ }^{1}$, The morbidity, mortality and economic burden associated with all types of URIs have been demonstrated significant, with influenza virus being the focus of identification as a causative pathogen for URIs in ambulatory clinical settings. ${ }^{1,2}$ Traditional diagnostic testing methods for URIs including antigenic methods, cell culture, and serology have limitations with regard to sensitivity, specificity, and/or turn-around-times, rendering them relatively limited for routine use in ambulatory care settings. ${ }^{3-5}$

Recent technological advances have led to the development of multiplexed molecular amplification assays that are capable of detecting multiple common causes of URI pathogens from a single nasopharyngeal swab 
(NP). Although these methods have been shown to be rapid, highly sensitive, and specific, ${ }^{6-9}$ uptake for routine practice remains relatively limited to research studies, due in part to lack of available treatment options for non-influenza respiratory viruses and added expense of employing multiplex molecular methods. ${ }^{6}$ On the other hand, these assays afford new opportunities to better understand the etiologic distribution, prevalence, and rates of co-infections associated with URIs. Several recent studies employing multiplex technologies have been conducted with specific select populations, including patients with community-associated pneumonia (CAP),${ }^{10-12}$ hospitalized patients, ${ }^{10-14}$ military personnel, ${ }^{15-17}$ relatively healthy outpatients ${ }^{7,}$ 18-20 and selected pediatrics cohorts. ${ }^{21-23}$

To date, there is limited data regarding the etiology of non-influenza, non-RSV, URI viral and bacterial pathogens in unselected ambulatory populations considered at high risk for respiratory virus related complications. Such research could be helpful not only for understanding the epidemiology and etiology of acute URIs, but also could help inform future research to address antibiotic stewardship. ${ }^{7}, 22,23$

Our aim was to contribute to the existing knowledge regarding the epidemiology and etiology of acute URIs in a broad ambulatory population of patients considered to be at high-risk of influenza complications, ${ }^{24,} 25$ who were tested for influenza, but were not tested for other respiratory pathogens. We collected residual waste samples from patients who presented to 4 geographically disparate EDs, who had already been tested for influenza with a single target influenza assay ${ }^{24}$ and subsequently tested them utilizing the multiplex Genmark ePlex respiratory panel (RP) research use only (RUO) platform.

\section{Methods}

\section{Study design}

Adults at high risk for influenza complications according to the Centers for Disease Control and Prevention (CDC) definition ${ }^{24}$ reporting to four U.S. EDs (Johns Hopkins Hospital, Baltimore, MD (JHH), Truman Medical Center, Kansas City, MO (TMC), Maricopa Medical Center, Phoenix, AZ (MMC), and Olive ViewUCLA Medical Center, Sylmar, CA (OMC)) were systematically screened by trained research coordinators, who assessed consecutive ED patients. All adult patients (age [?]18 years) were assessed for the presence of fever and/or respiratory symptoms, including documented fever (defined as $>100.4^{\circ} \mathrm{F}$ ) measured in the $\mathrm{ED}$ and any of the following, self-reported symptoms beginning within the previous 7 days: subjective fever, cough, nasal congestion, sinus congestion, rhinorrhea, sore throat, or shortness of breath. A patient who reported 1 or more of the above complaints was further evaluated to determine whether he or she met at least 1 of the 2011 CDC high-risk for influenza complication criteria for antiviral medication. ${ }^{24,}{ }^{25}$ Those patients, who met the CDC criteria for influenza antiviral treatment, spoke English, had not had a diagnosis of influenza within the last 2 weeks, had not been previously enrolled, and had the ability for follow-up were offered participation in the study and signed written consent forms. The Institutional Review Boards at each site approved the study protocol.

Clinical specimen and data collection

NPs were collected by trained clinical coordinators. Specimens were transported immediately to the laboratory in viral transport media, aliquoted, and stored at $-80^{\circ} \mathrm{C}$. Clinical data were collected by the trained clinical coordinators from patient questionnaires, and review of the electronic health record (EHR).

\section{Molecular detection of respiratory pathogens}

NPs underwent testing for influenza virus utilizing Prodesse ProFlu+ (Hologic, Bedford, MA) according to manufacturer instructions. A total of $41 \%(799 / 1941)$ had sufficient residual volumes to permit further testing with the ePlex RP RUO cartridge (Genmark Diagnostics, Carlsbad, CA). This multiplex assay detects: adenovirus (AdV); coronavirus HKU1, NL63, OC43, 229E, MERs (CoV); human metapneumovirus (hMPV); influenza A, A/H1N1, A/H1N1pdm 2009, A/H3N2 (IAV); influenza B (IBV), parainfluenza 14 (PIV), rhinovirus/enterovirus (RhV/EV), RSV A/B (RSV), Bordetella pertussis, Chlamydia pneumoniae ,Legionella pneumophila, Mycoplasma pneumoniae (M. pneumo). Testing was performed per manufacturer's instructions. Briefly, $200 \mu \mathrm{l}$ of NP was added to the specimen delivery device (SDD), vortexed for 10 seconds 
and added to the RP RUO cartridge. RP RUO cartridges were run on the ePlex platform. The complete assay time was 1 hour and 40 minutes.

Statistical analysis

Categorical variables were analyzed by Fisher's exact using R software and GraphPad Prism v 8.0.1. and a $p$ value of $<0.05$ was considered significant.

\section{Results}

Molecular detection of respiratory pathogens

The ePlex results demonstrated that 30.1\% (241/799) of patients tested positive for any respiratory pathogen, with $2 \%$ of the total specimens having co-infections (16/799) (Fig 1A). The composition of different pathogens detected is summarized in Figure 1B. RhV/EV was the most common pathogen detected $(32.7 \%$ of the total detections: mono- or co-infections), followed by IAV (26.1\%), CoV and hMPV (both 10.9\%), and RSV (9.3\%). Overall, $28.4 \%(69 / 241)$ of positives were infected with influenza (IAV or IBV). Less common pathogens were AdV (3.5\%), PIV (3.1\%), IBV (2.3\%), and M. pneumo (1.2\%).

Sixteen patients had co-infections (32 detections total), the composition of which is shown in Fig $1 \mathrm{~B}$ and C. IAV $(7 / 16,43.8 \%), \operatorname{RhV} / \mathrm{EV}(7 / 16,43.8 \%)$, and $\operatorname{AdV}(6 / 16,37.5 \%)$ were the most frequently detected pathogens in individuals with co-infections. For individuals infected with $\mathrm{AdV}$, co-infections $(\mathrm{N}=6,66.6 \%)$ were more common than mono-infections $(\mathrm{N}=3,33.3 \%)$, ratio of 2.0 (Fig $1 \mathrm{~B})$.

Geographical analysis

The prevalence of pathogens at the four sites were compared (Figure 2). The lowest rate of any pathogen detection was seen at JHH; 22.9\% (60/262) of specimens positive (Fig 2A). This was significantly different than those seen at MMC, 39.3\% (59/150, p =0.007, Fig 2B), TMC 31.5\% (53/168, p =0.003, Fig 2C), and OVM 31.5\% (69/219, p = 0.04, Fig 2D). Co-infections were most commonly found at MMC: $5.3 \%$ of specimens compared to approximately $1 \%$ at other sites.

All respiratory pathogens detected were observed at each site, with the exception of M. pneumoniae $(\mathrm{N}=2)$, which was only detected at MMC (Fig 2G). While not statistically significant, other differences were observed in the composition of pathogens between sites (Fig 2E-H). MMC had the greatest number of unique pathogens detected. RSV was the greatest at TMC (14.9\%). hMPV was identified in greater numbers and proportion at the Western EDs (14.9\% at MMC and $12.7 \%$ at OVM) compared to the Eastern ED (6.3\% at JHH).

Temporal analysis

Specimens were collected from November-April 2013-2014, the traditional time period for influenza/respiratory virus season. Graphing pathogens with $>20$ total detections (CoV, hMPV, IAV, and $\mathrm{RhV} / \mathrm{EV}$ ) across the 4 sites, temporal differences at the sites were observed (Fig 3). Trends in RhV/EV detection at all 4 sites were consistent over time. CoV, hMPV, and IAV showed similar trends at JHH, MMC, and OVM, but were distinct at TMC. TMC had an early IAV peak and later peaks of CoV and hMPV than the other sites.

\section{Patient characteristics}

Patient characteristics are described in Table 1. Briefly, a total of 799 specimens from 799 unique patients were analyzed. The range of ages was 18 to 93 years of age, median was 50 years and $60.6 \%(484 / 799)$ were females. $67.3 \%(538 / 799)$ had more than one condition of high-risk for influenza complication criteria, with the median being 2 conditions. Comparisons of patient demographics and outcomes with no detection, influenza detection, or other respiratory pathogen detections are shown in Table 1. While results were not statistically significant, there was a trend for a greater number of influenza-positive patients being admitted to the ICU and having radiographic diagnosis of pneumonia, compared to those with no pathogen or other pathogen. 


\section{Conclusions}

The recent emergence of SARS-CoV-2 (COVID-19) has demonstrated the important of surveillance for noninfluenza respiratory viruses. Here we present descriptive findings from non-influenza surveillance and how it can provide meaningful information regarding their composition and prevalence. Our goal was to add to the knowledge generated by other studies ${ }^{7,10-23}$ regarding viral etiologies associated with URIs aside from influenza viruses in a population of adults characterized as high-risk for influenza complications.

A few studies focusing on high-risk populations have employed multiplex molecular methods. For example, one study illustrated that there was a high incidence of complications related to non-influenza respiratory viruses, and that disease severity was similar to influenza, indicating that surveillance of these viruses is important. ${ }^{10}$ A large longitudinal retrospective study highlighted that picornaviruses, specifically $\mathrm{RhV} / \mathrm{EV}$, are potentially neglected as a significant contributor to the development of disease severity and can lead to lower respiratory infections. ${ }^{14}$ Lastly, other studies utilizing multiplex molecular methods have highlighted the importance of coinfections ${ }^{15}$ and diversity of non-influenza respiratory viruses. ${ }^{17}$

In this population of adults at high-risk for influenza complications, we identified a viral or bacterial respiratory pathogen etiology in 30.1\% (241/799) of the total specimens with a co-infection detection rate of $6.6 \%$ (16/241). Overall, the pathogens identified, AdV, CoV, hMPV, IV, PIV, RhV/EV, RSV and M. pneumo, are consistent with known causes of URIs, regardless of the specific population. ${ }^{26}$ We found that RhV/EV was the most common single pathogen detected in high-risk adult ED patients, and other studies have presented similar findings, albeit in military personnel. ${ }^{16,17}$

We found that AdV was more commonly associated with coinfections and this finding is supported by previous studies in military recruits ${ }^{15}$ and a study in hospitalized children that suggested that AdV may play a larger role than previously thought in the development of more severe disease, such as bronchitis and pneumonia. ${ }^{22}$ The pathogen detection rate and time of year for varied across the sites. The lowest detection rate was observed at JHH, while the highest detection (both mono- and co-infections) observed at MMC. MMC also had the greatest pathogen diversity and was the only site where atypical bacteria were found. While the $\mathrm{RhV} / \mathrm{EV}$ proportion of pathogens was fairly consistent across the sites, the peak detections for pathogens varied based on time of year. TMC had an early peak of influenza activity, with shifted peak detections of $\mathrm{CoV}$ and hMPV observed as compared to the other three sites. Similar results, in terms of temporal variation have been observed in other studies and each of these studies ${ }^{15,20,22}$ illustrated the temporal nature of respiratory virus infections. Situational awareness from broad surveillance may impact patient management.

This study had several limitations. First, it was only conducted over a single season and the numbers of positive tests was too low to make major comparisons. Additionally, because the population had many underlying conditions, there were not many differences found in the clinical outcomes and many confounders exist. Larger scale studies would be needed to make major conclusions on the impact of multiplex methods. However, this study does provide a description of the variety of pathogens found in adults at high-risk of influenza infections who report with influenza symptoms to the ED.

The value and meaningfulness of applying multiplex molecular methods for respiratory viruses aside from influenza virus has been under debate. ${ }^{27,28}$ For influenza virus, and in some cases RSV, studies utilizing point-of-care (POC) diagnostic tests for these specific organisms have shown value in terms of patient management, patient cohorting and droplet precautions, and appropriate anti-viral therapy. ${ }^{29-31}$ However, for large, molecular multiplex methods, it is unclear whether or not they should be employed broadly for general surveillance purposes, or restricted to specific populations, i.e., pediatric patients, high risk ED patients, and the immunocompromised. ${ }^{27}$ Coinfections are more readily detected on molecular panels, however, clinical consequences of coinfections are not totally clear. ${ }^{27}$ It has been noted that there are various barriers to utilizing these methods for general surveillance and specific diagnosis. These include difference in testing phase requirements, ${ }^{28}$ cost to the laboratory, the patient and insurer, ${ }^{27}$ and level of complexity for the multiplex method. ${ }^{27}$ Strategies have been suggested to mitigate some of these barriers. ${ }^{28}$ Surveillance of non-influenza 
viruses in these populations can aid in differentiating the cause of illness, which can be potentially antibiotic sparing. ${ }^{7}, 13,18-20$ Newly developed multiplex diagnostic technologies can aid in non-influenza surveillance and expand the etiology of URIs beyond influenza. ${ }^{7}, 18-20$

Overall, the applicability, clinical value and value to surveillance efforts of employing multiplex molecular methods may be specific to certain populations, such adults at high-risk for influenza complications, or may be of more benefit when applied locally, to identify small outbreaks of specific viruses not routinely surveyed for, in specific location.

\section{References}

1. Berry M, Gamieldien J, Fielding BC. Identification of new respiratory viruses in the new millennium. Viruses. 2015; 996-1019.

2. Fendrick AM, Monto AS, Nightengale B, Sarnes M. The economic burden of non-Influenza-related viral respiratory tract infection in the United States. Arch Intern Med. 2003; 487-94.

3. van Elden LJ, van Kraaij MG, Nijhuis M, Hendriksen KA, Dekker AW, Rozenberg-Arska M, van Loon AM. Polymerase chain reaction is more sensitive than viral culture and antigen testing for the detection of respiratory viruses in adults with hematological cancer and pneumonia. Clin Infect Dis. 2002 Jan $15 ; 34(2): 177-83$.

4. Mahony JB. Nucleic acid amplification-based diagnosis of respiratory virus infections. Expert Rev Anti Infect Ther. 2010; 1273-92.

5. Mahony JB, Petrich A, Smieja M. Molecular diagnosis of respiratory virus infections. Crit Rev Clin Lab Sci. 2011; 217-49.

6. Zimmerman RK, Rinaldo CR, Nowalk MP, Balasubramani GK, Moehling KK, Bullotta A, et al.Viral infections in outpatients with medically attended acute respiratory illness during the 2012-2013 Influenza season. BMC Infect Dis. 2015;15:87.

7. Green DA, Hitoaliaj L, Kotansky B, Campbell SM, Peaper DR. Clinical Utility of On-Demand Multiplex Respiratory Pathogen Testing among Adult Outpatients. J Clin Microbiol. 2016 Dec;54(12):29502955.

8. Chan M, Koo SH, Jiang B, Lim PQ, Tan TY. Comparison of the Biofire FilmArray Respiratory Panel, Seegene AnyplexII RV16, and Argene for the detection of respiratory viruses. J Clin Virol. 2018 Sep;106:13-17.

9. Babady NE, England MR, Jurcic Smith KL, He T, Wijetunge DS, Tang YW, Chamberland RR, Menegus M, Swierkosz EM, Jerris RC, Greene W. Multicenter Evaluation of the ePlex Respiratory Pathogen Panel for the Detection of Viral and Bacterial Respiratory Tract Pathogens in Nasopharyngeal Swabs. J Clin Microbiol. 2018 Jan 24;56(2).

10. Zhou F, Wang Y, Liu Y, Liu X, Gu L, Zhang X, Pu Z, Yang G, Liu B, Nie Q, Xue B, Feng J, Guo Q, Liu J, Fan H, Chen J, Zhang Y, Xu Z, Pang M, Chen Y, Nie X, Cai Z, Xu J, Peng K, Li X, Xiang P, Zhang Z, Jiang S, Su X, Zhang J, Li Y, Jin X, Jiang R, Dong J, Song Y, Zhou H, Wang C, Cao B; and the CAP-China Network. Disease severity and clinical outcomes of community-acquired pneumonia caused by non-influenza respiratory viruses in adults: a multicentre prospective registry study from the CAP-China Network. Eur Respir J. 2019 Aug 1;54(2).

11. Lim YK, Kweon OJ, Kim HR, Kim TH, Lee MK. Impact of bacterial and viral coinfection in community-acquired pneumonia in adults. Diagn Microbiol Infect Dis. 2019 May;94(1):50-54.

12. Quah J, Jiang B, Tan PC, Siau C, Tan TY. Impact of microbial Aetiology on mortality in severe community-acquired pneumonia. BMC Infect Dis. 2018 Sep 4;18(1):451. doi: 10.1186/s12879-018$3366-4$.

13. Çağlayan Serin D, Pullukçu H, Ciçek C, Sipahi OR, Taşbakan S, Atalay S; Pneumonia Study Group. Bacterial and viral etiology in hospitalized community acquired pneumonia with molecular methods and clinical evaluation. J Infect Dev Ctries. 2014 Apr 15;8(4):510-8.

14. Visseaux B, Burdet C, Voiriot G, Lescure FX, Chougar T, Brugière O, Crestani B, Casalino E, Charpentier C, Descamps D, Timsit JF, Yazdanpanah Y, Houhou-Fidouh N. Prevalence of respiratory viruses among adults, by season, age, respiratory tract region and type of medical unit in Paris, France, from 
2011 to 2016. PLoS One. 2017 Jul 14;12(7):e0180888.

15. Ho ZJ, Zhao X, Cook AR, Loh JP, Ng SH, Tan BH, Lee VJ. Clinical differences between respiratory viral and bacterial mono- and dual pathogen detected among Singapore military servicemen with febrile respiratory illness. Influenza Other Respir Viruses. 2015 Jul;9(4):200-8.

16. Lau YF, Koh WV, Kan C, Dua PA, Lim AE, Liaw CJ, Gao QH, Chng J, Lee VJ, Tan BH, Loh JP. Epidemiologic analysis of respiratory viral infections among Singapore military servicemen in 2016. BMC Infect Dis. 2018 Mar 12;18(1):123.

17. Tavakoli A, Karbalaie Niya MH, Bokharaei-Salim F, Farahmand M, Izadi M, Dorostkar R, Keyvani $\mathrm{H}$. The molecular epidemiology of respiratory viruses in military trainees in Iran. Med J Islam Repub Iran. 2019 May 8;33:40. doi:10.34171/mjiri.33.40. eCollection 2019.

18. Galanti M, Birger R, Ud-Dean M, Filip I, Morita H, Comito D, Anthony S, Freyer GA, Ibrahim S, Lane B, Ligon C, Rabadan R, Shittu A, Tagne E, Shaman J. Longitudinal active sampling for respiratory viral infections across age groups. Influenza Other Respiratory Viruses. 2019 Feb 15. doi: 10.1111/irv.12629. [Epub ahead of print]

19. Kaku N, Hashiguchi K, Iwanaga Y, Akamatsu N, Matsuda J, Kosai K, Uno N, Morinaga Y, Kitazaki T, Hasegawa H, Miyazaki T, Fukuda M, Izumikawa K, Mukae H, Yanagihara K. Evaluation of FilmArray respiratory panel multiplex polymerase chain reaction assay for detection of pathogens in adult outpatients with acute respiratory tract infection. J Infect Chemother. 2018 Sep;24(9):734-738.

20. Busson L, Bartiaux M, Brahim S, Konopnicki D, Dauby N, Gérard M, De Backer P, Van Vaerenbergh K, Mahadeb B, Mekkaoui L, De Foor M, Wautier M, Vandenberg O, Mols P, Levy J, Hallin M. Contribution of the FilmArray Respiratory Panel in the management of adult and pediatric patients attending the emergency room during 2015-2016 influenza epidemics: An interventional study. Int J Infect Dis. 2019 Jun;83:32-39.

21. Assane D, Makhtar C, Abdoulaye D, Amary F, Djibril B, Amadou D, Niokhor DJB, Amadou D, Cheikh L, Ndongo D, Mbayame N, Lamine F, Bouh BCS. Viral and Bacterial Etiologies of Acute Respiratory Infections Among Children Under 5 Years in Senegal. Microbiol Insights. 2018 Feb 13;11:1178636118758651

22. Kenmoe S, Tchendjou P, Vernet MA, Moyo-Tetang S, Mossus T, Njankouo-Ripa M, Kenne A, Penlap Beng V, Vabret A, Njouom R. Viral etiology of severe acute respiratory infections in hospitalized children in Cameroon, 2011-2013. Influenza Other Respir Viruses. 2016 Sep;10(5):386-93.

23. Finianos M, Issa R, Curran MD, Afif C, Rajab M, Irani J, Hakimeh N, Naous A, Hajj MJ, Hajj P, El Jisr T, El Chaar M. Etiology, seasonality, and clinical characterization of viral respiratory infections among hospitalized children in Beirut, Lebanon. J Med Virol. 2016 Nov;88(11):1874-81.

24. Dugas AF, Hsieh YH, Lovecchio F, Moran GJ, Steele MT, Talan DA, Rothman RE; ED National Influenza Network Investigators. Derivation and Validation of a Clinical Decision Guideline for Influenza Testing in Four U.S. Emergency Departments. Clin Infect Dis. 2020 Jan 1;70(1):49-58..

25. Kumar A, Zarychanski R, Pinto R, Cook DJ, Marshall J, Lacroix J, Stelfox T, Bagshaw S, Choong K, Lamontagne F, Turgeon AF, Lapinsky S, Ahern SP, Smith O, Siddiqui F, Jouvet P, Khwaja K, McIntyre L, Menon K, Hutchison J, Hornstein D, Joffe A, Lauzier F, Singh J, Karachi T, Wiebe K, Olafson K, Ramsey C, Sharma S, Dodek P, Meade M, Hall R, Fowler RA; Canadian Critical Care Trials Group H1N1 Collaborative. Critically ill patients with 2009 Influenza A(H1N1) infection in Canada. JAMA. 2009 Nov 4;302(17):1872-9.

26. Grief SN. Upper respiratory infections. Prim Care. 2013 Sep;40(3):757-70. doi:10.1016/j.pop.2013.06.004. Epub 2013 Jul 12. Review.

27. Schreckenberger PC, McAdam AJ. Point-Counterpoint: Large Multiplex PCR Panels Should Be First-Line Tests for Detection of Respiratory and Intestinal Pathogens. J Clin Microbiol. 2015 Oct;53(10):3110-5.

28. Diaz-Decaro JD, Green NM, Godwin HA. Critical evaluation of FDA-approved respiratory multiplex assays for public health surveillance. Expert Rev Mol Diagn. 2018 Jul;18(7):631-643.

29. Pedersen CJ, Rogan DT, Yang S, Quinn JV. Using a novel rapid viral test to improve triage of emergency department patients with acute respiratory illness during flu season. J Clin Virol. 2018 
Nov;108:72-76.

30. Benirschke RC, McElvania E, Thomson RB Jr, Kaul KL, Das S. Clinical Impact of Rapid Point-of-Care PCR Influenza Testing in an Urgent Care Setting: a Single-Center Study. J Clin Microbiol. 2019 Feb $27 ; 57(3)$.

31. Rahamat-Langendoen J, Groenewoud H, Kuijpers J, Melchers WJG, van der Wilt GJ. Impact of molecular point-of-care testing on clinical management and in-hospital costs of patients suspected of influenza or RSV infection: a modeling study. J Med Virol. 2019 Aug;91(8):1408-1414.

Table 1. Patient characteristics across all sites

\begin{tabular}{ll}
\hline & Total \\
\hline $\mathrm{N}$ & 799 \\
$\begin{array}{l}\text { Demographics } \\
\text { Age }\end{array}$ & $50(18-93)$ \\
Gender & $484(60.6 \%)$ \\
Female & \\
Ethnicity & $259(32.7 \%)$ \\
Hispanic or Latino & \\
Race & $348(43.6 \%)$ \\
Black & $199(24.9 \%)$ \\
White & $10(1.3 \%)$ \\
Asian & $12(1.5 \%)$ \\
American Indian & $224(28.0 \%)$ \\
Other & \\
CDC high risk & $538(67.3 \%)$ \\
Greater than 1 & \\
Disease severity & $214(26.8 \%)$ \\
Oxygen supplementation & $348(43.6 \%)$ \\
Admitted & $3(.5-29)$ \\
Hospital length of stay (days) & $45(13 \%)$ \\
ICU & $3(1-21)$ \\
ICU length of stay (days) & $158(19.8 \%)$ \\
Pneumonia & $1(0 \%)$ \\
Death & Values shown as N (\%). For length of stay = median (range \\
Values shown as N (\%). For length of stay = median (range) &
\end{tabular}

Figure 1. Detection of pathogens. A. Total detections in the 799 specimens. Blue $=$ mono-infections and red $=$ co-infections.B. Specific pathogens detected. Graph shows percent detected in mono- (blue) versus co-infections (red). The total number of detections $(\mathrm{N})$ for each pathogen (inclusive of mono- and co-infections).C. 16 specimens had co-infections detected (32 pathogens total). The heatmap shows the number of co-infections. Each pathogen is plotted with all other pathogens detected from the 799 specimens. Number of co-infections detected white $=0$, grey $=1$, black $=2$, red $=3$.

Figure 2. Geographic analysis. A-D. Detections at each site. A. Johns Hopkins Hospital (JHH), B. Truman Medical Center (TMC), C. Maricopa Medical Center (MMC), D. Olive-View UCLA Medical (OVM). Top pie charts show total detections broken down by mono-infections (blue) and co-infections (red). Bottom pie charts show the distribution of pathogens detected at each site.

Figure 3. Temporal analysis. Graphs show each pathogen that had N $>20$ detections across all 4 sites. $\mathrm{X}$-axis is time, $\mathrm{Y}$-axis is \% of the total pathogens. JHH $=$ blue lines, $\mathrm{TMC}=$ red lines, $\mathrm{MMC}=$ black lines, and $\mathrm{OVM}=$ purple lines 
A

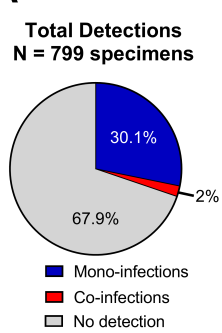

B

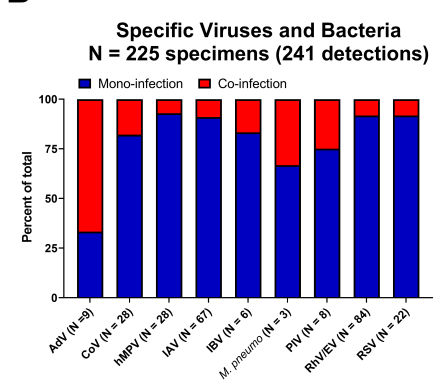

C Co-infections ( $\mathrm{N}=16$ specimens)

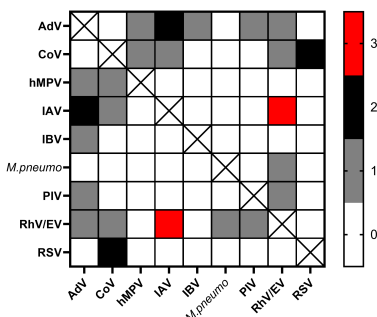
A $\underset{\mathrm{NHH}}{\mathrm{JH}}=\mathbf{2 6 2}$

B

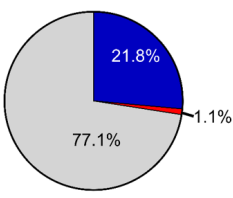

B $\begin{gathered}\text { TMC } \\ N=168\end{gathered}$

C

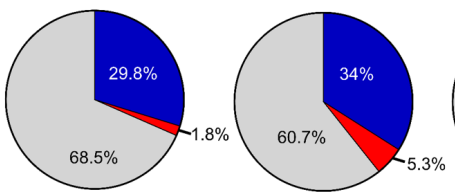

OVM
$\mathbf{N}=\mathbf{2 1 9}$
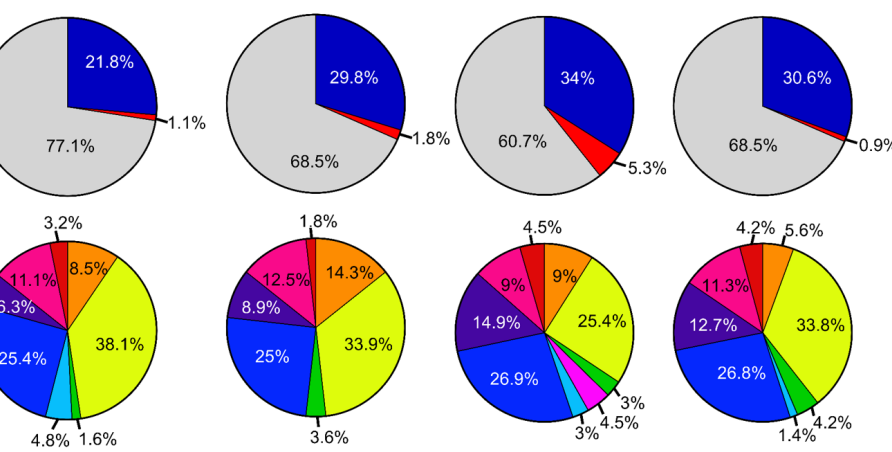

Detections

Mono-infections Co-infections $\square$ No detection

CoV

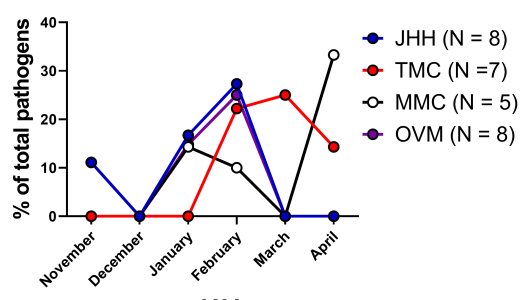

IAV

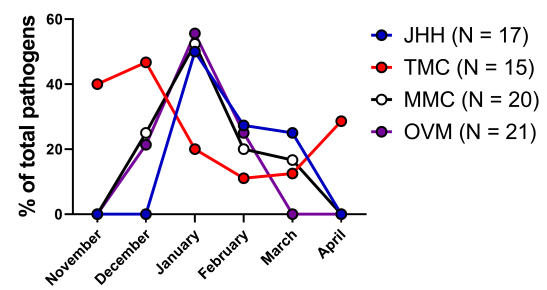

hMPV

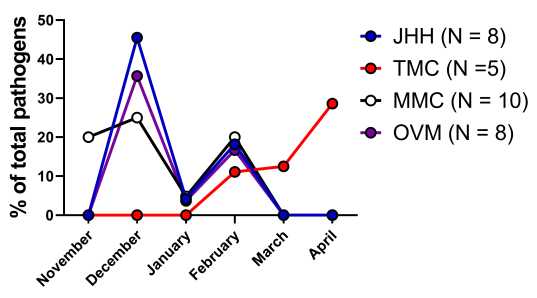

RhV/EV

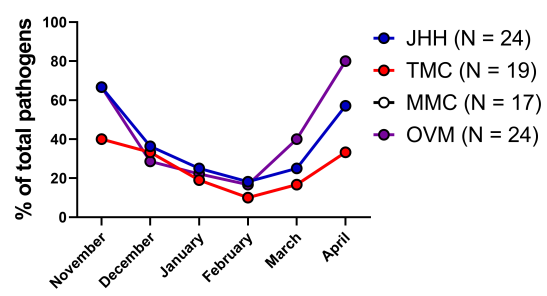

